\title{
IL CONCETTO DI STORIA DELLA LINGUA NELL'OPERA GRAMMATICALE DI BENEDETTO BUOMMATTEI
}

Che la storia della lingua sia una disciplina linguistica di data recente, fondata nell' 800 , soprattutto grazie alle ricerche pionieristiche di insigni filologi tedeschi, è un'opinione comunemente accettata, ed essa è probabilmente anche giustificata attraverso la classificazione datane di disciplina. A questo dato di fatto si deve senz'altro l'opinione altrettanto estesa che prima dell' 800 non esistesse il concetto di linguistica diacronica o cambiamento linguistico in senso scientifico, come pure quella non meno erronea che la linguistica, come scienza, sia stata fondata solo nell'800.

Nonostante il crescente interesse degli ultimi decenni per la storia della linguistica, il riconoscimento di radici più remote della linguistica e della storia della lingua non è ancora diventato comune nell'ambiente dei linguisti moderni. Infatti, anche prima di Bopp e Diez (per la storia della lingua) e di De Saussure (per la linguistica), si sono svolte delle attività linguistiche non trascurabili: anzi, molti concetti della linguistica moderna, ritenuti di data recente, risalgono a studiosi dei secoli passati, e per capire a fondo le implicazioni di tali concetti, bisogna studiarli nella prospettiva storica. Quindi, i pochi studiosi che si occupano di storia della linguistica hanno un importante messaggio da trasmettere ai linguisti di oggi.

Già nel Rinascimento la nozione di cambiamento linguistico era oggetto di riflessioni linguistiche, come hanno dimostrato tra l'altro Hall (1936), Faithfull (1962) e Simone (1976). Ma a parte le eccezioni di queste ricerche, gli studi sui lavori grammaticali del Rinascimento si rivolgono piuttosto all'aspetto filologico della questione della lingua (Simone, 1976, 302).

Se ho scelto di trattare tale problematica a partire dall'opera di Benedetto Buommattei, non è, come si potrebbe supporre, perché essa segni rinnovamenti eccezionali nel modo di considerare la storia della lingua rispetto alla tradizione precedente o rispetto agli studiosi contemporanei. Studiato sotto questo aspetto, il Buommattei - che per altri versi rivela veri pregi di progressista nel campo della grammatica (scienziato contemporaneo, anche spiritualmente, di Galilei) - risulta piuttosto tradizionale, a parte singoli dettagli, come vedremo oltre. Sono invece la chiarezza con cui il Buommattei espone le sue idee, nonché il periodo postrinascimentale e l'ambiente della "nuova scienza", a renderlo idoneo come punto di partenza. 
Con il procedimento scelto vorrei inoltre ribadire la necessità di studiare il contributo del singolo linguista (o scienziato in genere) non come fenomeno isolato, il che può indurre a non ben fondate conclusioni intorno alla sua originalità, ma visto in relazione alla tradizione di cui fa parte, da cui dipende il suo atteggiamento e su cui si costruisce il suo universo.

Benedetto Buommattei (1581 - 1647), scienziato, “pubblico lettore della lingua toscana nello studio pisano e fiorentino“, segretario dell'Accademia della Crusca, insigne teologo ecc., è autore dell'opera linguistica e grammaticale Della lingua toscana, Vol. I-II. Il primo volume, che fu pubblicato nel 1623, è quello più importante in senso teorico, in quanto espone le idee del Buommattei sulla linguistica generale, basate su principi razionalistici e universalistici. Da notare che questo lavoro precede di quasi $\mathbf{4 0}$ anni quello più famoso di Arnaut e Lancelot, Grammaire générale et raisonnée, Paris, 1660 . Il secondo volume, che applica le idee generali alla lingua toscana, esce nel 1643. Nello stesso anno di pubblicazione del primo volume, Benedetto Buommattei "recita pubblicamente nell'Accademia Fiorentina" un discorso intitolato Delle lodi della lingua toscana. Questo discorso è compreso nella 6.a edizione dell'opera grammaticale del 1807 (v. la bibliografia), alle pp. 541-568. È soprattutto quest'ultimo testo che fornisce la base per la seguente esposizione, completata secondo il bisogno con brani tratti dal Vol. I, 1623.

Il titolo del discorso annuncia con la parola lodi un argomento centrale nelle riflessioni linguistiche del dibattito corrente: la questione del prestigio di una data lingua. Il Buommattei era un fervente propugnatore della lingua toscana, e tutto il discorso è una ponderata argomentazione in favore del prestigio, ossia della nobiltà o dignità, della lingua toscana contemporanea.

Partendo dal suo obiettivo principale, e cioè di dimostrare la dignità della lingua toscana, il Buommattei distingue i fattori che possono conferire prestigio alla lingua in due tipi: 1) fattori esterni e comuni (origine, uso da parte del popolo e da parte degli scrittori, condizioni climatiche e posizione geografica); 2) proprieta particolari e interne (in termini moderni: funzionalità della struttura linguistica), adoperando una terminologia che corrisponde a quella odierna: 'external', 'internal history'/ 'Externe', 'Interne Sprachgeschichte'.

Tra i fattori esterni viene considerata prima di tutto l'origine. Già l'idea di riflettere sull'origine implica un approccio storico allo studio della lingua. Dell'origine dell'italiano si era discusso già dall'Umanesimo, periodo in cui era cominciato a farsi più insistente il bisogno di considerare l'italiano come una lingua per sé, indipendente, soprattutto per giustificare il suo uso scritto per lavori letterari e filosofici, e non come una specie di registro informale del latino (Skytte, 1990, p. 270). Nel Medioevo il latino, probabilmente, veniva considerato come la lingua (e cosi anche grammatica equivaleva a 'latino'). 
Non può sorprendere nel caso dell'italiano che il latino normalmente venisse supposto come lingua d'origine: infatti, le prime discussioni (e qui prescindiamo dal De vulgari Eloquentia di Dante, che non era noto ai contemporanei) intorno all'origine (Tavoni, 1986) vertevano sulla questione di quale latino. Ma con la crescente conoscenza di altre lingue (nel ' 400 si era cominciati a studiare il greco, nel '500 l'ebraico e l'arabo, e con le scoperte geografiche ci si doveva rendere conto dell'esistenza di numerose lingue esotiche) apparirono nuove teorie, come quella, di ispirazione teologica, sull'origine ebraica non solo dell'italiano, ma di tutte le lingue (Keith Percival, 1986, p. 63), o quella sulla derivazione etrusca e aramea dell'italiano, esposta da Pierfrancesco Giambullari (1495-1555) in Il Gello. Ragionamenti de la prima ed antica origine della Toscana, et particolarmente della lingua Fiorentina (Coseriu, 1977, p. 104), idea del resto già lanciata da Niccolò della Luna nel '400 (Tavoni, 1986, p. 30).

Il Buommattei, fondandosi sul patrimonio lessicale, così esprime il suo parere a proposito:

“Ella riconosce in gran parte l'origine sua, come pare a più e più dotti, dalla Latina, e in buona parte ancora dalla Greca; le più nobili, le più pregiate, le più maestose, e le più venerande che sieno state giammai, eccettuatene solo l'Ebrea, che per li profondi misterj suoi, e per gli altri privilegi, di ch'è stata arricchita dal grande Iddio, penso che sdegnerebbe d'esser cimentata coll'altre..." (op. cit., p. 545).

L'idea dell'origine greca non era del tutto nuova, ed era dovuta al prestigio, nel Rinascimento, della lingua greca come lingua "perfetta". Anche per il francese era stata avanzata nel '500 l'ipotesi dell'origine greca (Brincat, 1986, p. 98).

Mentre il Buommattei chiaramente rifiuta la spiegazione teologica dell'origine della lingua, la sua argomentazione per la tesi dell'orgine greco-latina dell'italiano risulta assai debole, e poco scientifica. Scientifico è invece il tentativo ben più interessante di Celso Cittadini (1553-1627) di fornire una documentazione precisa dell'origine latina dell'italiano. Questa documentazione, pubblicata nel Della vera origine e del processo e nome della nostra lingua (Venezia, 1601), è basata su testi di autori latini e su iscrizioni antiche (Faithfull, 1962, pp. 243-255). Osservando le variazioni lessicali nei testi latini, il Cittadini se ne serve per esporre una teoria sull'esistenza nella Roma antica di un romano primitivo (= 'latino volgare' nella terminologia odierna) e di un tardo romano (cioè la lingua scritta, nobile = 'latino classico'). Per darne un'idea citiamo qualche esempio del lavoro del Cittadini:

\section{Romano primitivo}

coda

caballus

vostra

fructi
Tardo romano

cauda

equus

vestra

fructus 
In base a questi e molti altri esempi, confrontati poi con quelli corrispondenti del volgare italiano, il Cittadini dimostra che questo risale al romano primitivo. Interessantissime sono le testimonianze (cavate da testi antichi) di sviluppi fonetici, come p. es.

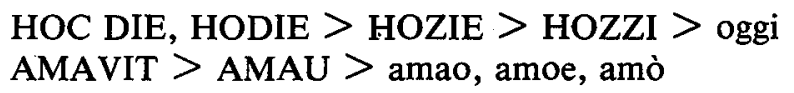

L'opera del Cittadini anticipa con tutta evidenza la grammatica storica dell' 800 .

Come abbiamo visto in Cittadini, l'idea dell'origine della lingua implica anche l'idea del mutamento. Nel discorso del Buommattei, l'origine nobile è considerata in favore del prestigio della lingua toscana. Tuttavia, una nobile origine non è necessariamente sufficiente a garantire la nobiltà della lingua derivata:

“... quand'e' si può provare, che una lingua derivi da un'altra nobile, e quella dovrà dirsi nobile, se già ella non digenerasse dalla sua ragguardevole origine.“ (op. cit., p. 544).

Il mutamento, dunque, può anche verificarsi come degenerazione. Infatti, quando nel '400 erano cominciate le discussioni sull'origine latina dell'italiano, il termine normalmente usato per designare il mutamento era stato quello di corruzione (conformemente a una teoria che spesso nella letteratura viene menzionata come 'teoria della catastrofe'). Con la crescita del prestigio del volgare, tale termine viene man mano sostituito con termini più neutri come generazione (p. es. Varchi) o alterazione (p. es. Castelvetro, Tolomei, Cittadini).

Accanto all'evolversi del concetto di mutamento, nasce il bisogno di interpretarne le cause. Per tutto il '500 l'interpretazione comunemente accettata era quella esposta da P. Bembo nelle sue Prose, sul mescolamento della lingua romana con quella o quelle dei Barbari. Claudio Marazzini (1989, p. 22 ss.) discute il lato negativo della teoria delle 'origini barbare': essa, infatti, "serviva perfettamente come argomento per i nemici dell'italiano. "Forse è addirittura questo il motivo che spinge il Giambullari ad avanzare la sua teoria sull'origine etrusca ed aramea della lingua toscana, per evitare così la 'teoria della catastrofe' (Marazzini, 1989, p. 26).

Benedetto Buommattei riprende il filo del discorso, affrontando da scienziato i vari elementi "penosi" della tradizione. Il suo punto di vista, molto più articolato, sul "valore" del mutamento, procede con chiarezza dal titolo del Capo III (Trattato Primo, Primo Libro del Della lingua toscana, pp. 99-104) Dove, quando, e come la lingua Toscana si generasse, crescesse, cascasse e risurgesse. Il capitolo comincia cosi :

"Per quel che da'più intendenti Scrittori (dalle conghietture più che da altro ajutati) possiam cavare, la lingua nostra, quanto al corpo naturale delle sue pa- 
role, ricevè i suoi primi principj dalla Latina, con altre straniere confusa. Il che se fosse dopo la incursion de'Barbari (come pare al Bembo) o pure sin quando cominciarono a dominare Imperatori stranieri e barbari (come disputa l'autor della Giunta [Castelvetro] basta dire che mescolamento di parlar forestiero con l'originario latino produsse una terza specie di lingua." (op. cit., p. 99).

Si noti la riserva, tipica dell'atteggiamento del Buommattei, a proposito della validità della teoria ("conghiettura"). Il "mescolamento" delle lingue genera o produce una terza lingua: qui, veramente, non si parla di corruzione. Al contrario, il Buommattei si volge a ribattere le connotazioni negative dei termini mescolamento e barbaro:

"E se alcuno vago d'opporsi a'miei detti, e alla grandezza di questa lingua, dicesse, che un miscuglio si fatto non può non averle portato detrimento notabile, e perciò esser caduta in gran parte da quella nobiltà, che le sarebbe conferita dalla latina, risponderei prontamente, che quando il mescolamento si fa con cosa d'inferior condizione, quel che si mescola può riceverne danno, come chi mette l'acqua o cosa sì fatta nel vino; ma quando vi si mette cosa di pari bontà, o migliore, ella può mutarsi e non peggiorare, e anche talora può guadagnarne, come chi mette il vino bianco nel rosso, o il greco nella verdea, o trebbiano, mutano e sapore e colore, e forse in parte ancora temperamento, ma non diventano cattivi.“ (Delle lodi, p. 549).

La metafora del vino mescolato sembra essere comune nella letteratura sulla questione della lingua, come del resto molte altre metafore adoperate a proposito della lingua. Si confronti p. es. il commento di Lazzaro Bonamico nel Dialogo di Sperone Speroni:

"tale sia la volgare thoscana rispetto alla lingua latina; quale la feccia al vino: peroche la volgare non è altro che la latina guasta e corrotta hoggimai dalla lunghezza del tempo, o dalla forza dei Barbari“ (Speroni, 1989, p. 168).

A proposito dei barbari, il Buommattei, rovesciando l'immagine tradizionale dei barbari, si pronuncia così:

"Nè si lasci alcuno ingannar da questo nome, che si dà loro di barbari, perchè noi in questo, come in molte altre cose, seguitiamo i Greci e i Latini, che chiamavan barbari tutti quelli, che avevan il parlar diverso da loro, quasi volesser con quella parola aspra e ruvida bar ba ro accennar contraffacendo, quella ruvidezza, che a loro pareva sentire in tutte le lingue straniere. Furono grandi e famosi que'barbari ... Nè mancò fra loro chi le buone arti, e le liberali discipline e favorisse, e abbracciasse; e in particular nelle leggi, e ne'governi molti di loro dimostraron ingegno e spirito più che ordinario...“ (op. cit., p. 550). 
Un altro tratto da notare nel titolo del Capo III è l'applicazione di una valutazione graduata dell'evoluzione della lingua toscana, della quale è detto che si genera, cresce, casca, e risurge. Con crescere il Buommattei si riferisce al periodo dei grandi Trecentisti, Dante, Petrarca e Boccaccio. Ma già dal 1350 la situazione cambia e segue il periodo della "cascata":

"E se il nuovo risurger che fece la lingua Latina intorno al 1350 (di che si dee non poca lode al Petrarca) non avesse per lo spazio di più di 150 anni interrotto il suo corso, si può creder ch'ella sarebbe ora a tal grado venuta a quale forse niun'altra potette arrivar giammai. Ma ... poco mancò ch'ella [la risurgente lingua Latina] non la riducesse al niente, della dignità della scrittura parlando... Questo fece, che non solamente gli scritti, ma il comun parlare eziandio se n'andò fino al 1500 peggiorando mai sempre." (op. cit., p. 193).

Dopo il 1500 , ecco il risurgere della lingua toscana, grazie a:

"gli uomini dietro alle pedate del Poliziano, del Bembo, del Casa, dell'Ariosto, e d'altri valorosi scrittori di que'tempi...“ (op. cit., p. 104).

Sul proprio periodo, il Buommattei si esprime con ottimismo:

“... e va tanto migliorando ogni giorno, ch'ella si vede camminare a gran passo a quell'antico grado di gloria ch'ell'era quando ne cadde;...“ (op. cit., p. 104).

Sembra che il fattore più importante per quanto riguarda l'evoluzione della lingua, come schizzata finora dal Buommattei, sia stato l'influsso degli scrittori. Però è interessante notare, anche rispetto ai pareri dei contemporanei, il peso attribuito ad altri fattori, come p.es. fattori di carattere sociolinguistico e influsso della lingua parlata.

Infatti, la teoria del "mescolamento" implica una interpretazione comunicativa. Secondo Raffaele Simone (1976, p. 307) già nella linguistica del '500 si può osservare "l'anticipation d'une attitude sociolinguistique", soprattutto manifesta nell'opera di Sperone Speroni Dialogo delle lingue (1530-35).

Il fattore comunicativo è ampiamente trattato dal Buommattei (op. cit. p. 100-101): tra latini e barbari, tra governo e popolo nelle repubbliche, e perfino tra innamorati (la poesia provenzale e siciliana); e in genere il Buommattei si riferisce costantemente alla lingua parlata di tutti i giorni, in qualche caso con distinzioni secondo le varie classi sociali. Al popolo viene attribuito un ruolo importante per lo sviluppo linguistico:

"il popolo è quel che forma le lingue, e le sue regole, almeno materialmente, e gli scrittori son que' che le raccolgono, e stabiliscono.“ (op. cit., p. 109). 
E lo stesso vale anche per i vocaboli:

“... molti se ne forman dal popolo tutto di, che ancora non sono stati registrati dagli scrittori...“ (op. cit., p. 109). dita:

Nell'affermazione seguente, tale importanza viene ulteriormente riba-

"E se la gramatica non è altro che una scienza di parlar per uso, potremo dir che quest'uso si debb'apprender dal popolo, come da autore, e padrone, e la scienza si convenga pigliar dagli scrittori, come da maestri, e interpreti. “(op. cit., p. 109).

Ai criteri sopra elencati, Buommattei ne aggiunge altri due, comuni nel periodo, cioè quello climatico e quello geografico, che servono per spiegare la variazione linguistica:

"Ma siccome la lingua latina o per lo diverso temperamento dell'aria, o per la più o meno vicinità o lontananza da Roma, soleva essere in tutte le regioni d'Italia o nella sustanza o negli accidenti molto diversa; così la nata lingua volgare ritenne le medesime, o poco dissimili differenze nelle stesse regioni; sentendosi pure tra l'un popolo e l'altro, come già s'è mostrato, diversità non piccola e ne'corpi delle parole, e nel modo del pronunziarle." (op. cit., p. 101). (NB: accidenti indica i morfemi flessivi ed i morfemi derivazionali).

Sul fattore climatico, il Buommattei ritorna ancora almeno due volte, accennando all" "aria temperatissima" della Toscana (p. 101) e, nelle Lodi - alla "salubrità dell'aria" (p. 567), fattore che però sembra di minima importanza. Per l'interpretazione climatica, attuale ancora nel '700, si confronti Marazzini (1989, p. 120).

Tutti i fattori esaminati finora per rilevare il prestigio della lingua toscana, sono quelli dal Buommattei denominati esterni:

"Però, concludendo, diremo, che se la nostra lingua riconosce, sì bella origine, quale è la Latina e la Greca; se è stata messa in uso da così famosi popoli, e preconizzata da Scrittori si degni; e se ha avuto principio sotto Ciel sì benigno; ella potrà dirsi per questo capo al pari di ogni altra nobile. (...) Ma... se ella non avesse altri pregi, ella non passerebbe al sicuro la mediocrità di molt'altre. “ (op. cit., p. 556).

E a questo punto segue un esame dei fattori interni. Ricordando il lato funzionale della lingua (che deve "giovare e servire"), il Buommattei sostiene che la nobiltà della lingua dipende prima di tutto dalla facilità, dalla chiarezza e dalla brevità con cui essa si presta a "palesare i concetti dell'uomo" (p. 558). La lingua toscana possie- 
de tali meriti "sopr'ogni credere" grazie a valori immanenti, come p. es. la ricchezza di vocaboli, i quali, poi, attraverso i suffissi possono assumere un'infinità di sfumature, nonché alla ricchezza di strutture sintattiche:

“... ella [la lingua toscana] può esplicar bene spesso in una sola parola quel che un'altra d'accidenti, e di frasi meno abbondante sarà forzata a descriver con tre o quattro.“ (op. cit., p. 559).

È interessante questa svolta nel decidere la causa della "nobiltà " o del prestigio della lingua toscana. Presso i grammatici del '500 (anche quelli preferiti dallo stesso Buommattei, come p.es. il Bembo e il Varchi) i criteri che determinano la nobiltà della lingua sono quelli cosiddetti esterni, e soprattutto quello degli "scrittori":

"Le lingue nobili non è dubbio che hanno mica l'esser, ma l'esser nobile (...) degli scrittori“" (Varchi).

Svolta, certo da mettere in rapporto con l'atteggiamento razionalistico del Buommattei, e che nello stesso tempo segna un cambiamento radicale nel modo di considerare il latino. Secondo il Buommattei, il latino non possiede pregi di brevità comparabili a quelli del toscano. Tra gli argomenti addotti in favore della lingua toscana rispetto al latino, citiamo:

"per variar in più maniere i suoi vocaboli ... la differenza che è tra ho amato e amai, e tra amerei e amassi...; sì per tesser la sua orazion di più parti, avendon'oltre all'otto de'Latini altre quattro, et in particolar l'articolo...; sì per essere abbondantissima di molte frasi, con le quali può allungare e abbreviar i periodi a suo talento. Ma quel, che per tutto vale, è, perchè in una sola parola spesso racchiude più parti, come sono andovvi, portommelo, e altri simili affissi;... (op. cit., p. 563).

Secondo i fautori del latino una prova della superiorità di questo sarebbe la lunghezza delle traduzioni in italiano, rispetto al testo latino originario. Ma questo, ribatte il Buommattei, è un fenomeno che vale per le traduzioni in genere, e rivolto al suo uditorio dell'Accademia Fiorentina, egli afferma:

"Ma non vi lasciate, o giovani, persuader tal fallacia, e rispondete pur loro arditamente, che la difficoltà del tradur d'un idioma in un altro sarà da loro provata, se essi tenteranno di tradur nel Latino dal nostro." (op. cit. p. 562).

In relazione al cambiato ruolo del latino, che si manifesta con tutta chiarezza nel testo del Buommattei, è significativo che gli aggettivi vivo e morto applicati a proposito di lingua fanno la loro prima apparizione proprio in questo periodo. Secondo il $D E L I$ la prima attestazione sarebbe presso Salviati nel 1586. Ma già nel Dialogo di Speroni (Bossong, 1990, 118) troviamo: "le vostre morte Latine parole ... le nostre vive volgari“. 
Nel discorso Delle lodi non vengono toccate questioni etimologiche. Ma dato che l'etimologia, ossia la coscienza di una "storia" della parola, costituisce una parte notevole della coscienza linguistica del tempo, esaminiamo brevemente questo fattore. Già nella Grammatichetta quattrocentesca, attribuita a L.B. Alberti, appaiono cenni sull'origine straniera, germanica, di certe parole toscane. $\mathrm{E}$ in questo contesto si può ricordare che la teoria del "mescolamento" è in gran parte basata sul riconoscimento dell'esistenza di parole "barbare" nel vocabolario italiano. Ma oltre a questo, dai tempi più remoti, l'etimologia, ossia l'origine, era sempre stata connessa con un certo fascino di carattere magico-teologico.

Nell'opera del Buommattei l'etimologia serve spesso per scopi didattici: introducendo un nuovo termine, il Buommattei ama servirsi dell'etimologia per dimostrare le sfumature semantiche del vocabolo e per definire il concetto. Così, p. es. nel presentare il termine lettera (a proposito della scrittura), egli passa in rassegna 5 possibili etimologie al lat. LITERA (LEGE ITERUM; LEGE IN ITINERE; LITURA 'macchia'; LITURA 'quia deleri potest'; LITERA: "quasi lineatura“), per poi concludere:

"Di maniera che da tutta questa varietà d'Etimologie, a me pare ch'e'si possa far sicuro argomento, che lettera nel suo proprio e stretto significato, si pigli per una parte della scrittura; poichè la lettera si fa di linee, la linea può cancellarsi, ma cancellar non si può, se prima non è formata; ed essendo formata in modo ch'ella possa leggersi, avrà sembiante di scrittura e non di pura favella." (op. cit., p. 132).

E si confronti inoltre il commento a proposito del termine verbo per designare la classe di parole:

“La nota delle azioni si chiama verbo, così detta già da'Latini, da'quali l'abbiam presa poi noi, credo per dimostrar la sua nobiltà; perchè dicendo essi generalmente tutte le parole verbum, dissero poi a questa in ispezie verbum, come se volessero dire, che questa sia di tutte l'altre più degna..." (Vol. I, p. 324).

Oltre alla coscienza della storia, rimane ancora presso il Buommattei una reminiscenza della "magia della parola".

Mentre nell'opera del Buommattei non si può parlare di innovazione per quanto riguarda l'etimologia, è da notare che i primi approcci scientifici all'etimologia risalgono al '600. Per l'italiano va ricordato l'impressionante lavoro dello studioso francese Gilles Ménage, che sotto il nome italianizzato di Egidio Menagio pubblicò un grosso vocabolario etimologico della lingua italiana Le origini della lingua italiana (Parigi 1669). La storia, complessa e intricata, di questo stupendo lavoro, che è di qualità e precisione scientifica sorprendenti, e finora poco notato, è ora trattato ampiamente da Marazzini (1989, p. 47 ss.). 
Rispetto al periodo del Buommattei, la storia della lingua oggi ha raggiunto un livello scientifico assai considerevole, come esemplificato nell'insigne opera di Pavao Tekavčić.

Tuttavia, nella concezione comune (benché non in quella del mondo degli scienziati) del mutamento linguistico sussistono molti dei vecchi pregiudizi, sulla "corruzione" della lingua e sulla superiorità di certe lingue rispetto ad altre. Da parte scientifica, già nella sua tesi per il dottorato, pubblicata in inglese nel 1894 sotto il titolo di Progress in Language, Otto Jespersen aveva confutato la teoria della "degenerazione" linguistica, a proposito dell'inglese. E per un contributo diretto a ribattere i pregiudizi sulla superiorità di una data lingua, in casu il francese, si può ricordare l'articolo di Pierre Swiggers La clarté du francais.

D'altra parte possiamo constatare che la coscienza scientifica di una storia della lingua ha radici remote. Sembra assurdo il tentativo di dare una data precisa all'inizio della storia della lingua come scienza. Nel caso dello studio della lingua italiana, già nel ' 500 e nel ' 600 ci sono, come abbiamo visto, esempi evidenti di un atteggiamento scientifico rispetto al cambiamento della lingua.

\section{BIBLIOGRAFIA}

Alberti, Leon Battista. 1964. La prima grammatica della lingua volgare. A c. di Cecil Grayson. Bologna.

Arnauld, Antoine et Lancelot, Claude. 1660. Grammaire générale et raisonnée. Paris.

Bembo, Pietro. 1955. Prose della volgar lingua. A c. di Mario Marti. Padova.

Bossong, Georg. 1990. Sprachwissenschaft und Sprachphilosophie in der Romania. Tübingen.

Brincat, Giuseppe. 1986. La linguistica prestrutturale. Bologna.

Buommattei, Benedetto. 1807. Della lingua toscana. Milano.

Cortelazzo, Manlio/ Zolli, Paolo. 1983. Dizionario etimologico della lingua italiana. Vol. 3. (DELI). Bologna.

Coseriu, Eugenio. 1977. Tradición y novedad en la ciencia del lenguaje. Estudios de historia de la lingüística. Madrid.

Droixhe, Daniel. 1978. La linguistique et l'appel de l'histoire (1600-1800). Genève.

Faithfull, R.G. 1962. Teorie filologiche nell'Italia del primo Seicento con particolare riferimento alla filologia volgare. In Studi di Filologia Italiana, XX, pp. 147-313.

Garin, Eugenio. 1965. L'umanesimo italiano. Bari.

Giambullari, Pierfrancesco. 1986. Regole della lingua fiorentina. A c. di Ilaria Bonomi. Firenze.

Hall, R.A. jr. 1936. Linguistic Theory in the Italian Renaissance. In Language, 13 , pp. $96-107$. 
Jespersen, Otto. 1894. Progress in Language. London.

Marazzini, Claudio. 1989. Storia e coscienza della lingua in Italia dall'umanesimo al romanticismo. Torino.

Menagio, Egidio. 1685. Le origini della lingua italiana. Genève.

Migliorini, Bruno. 1963. Storia della lingua italiana. Firenze.

Padley, G.A. 1985. 1988. Grammatical Theory in Western Europe 1500-1700. Trends in Vernacular Grammar. I-II. Cambridge.

Percival, W. Keith. 1986. Renaissance linguistics: the old and the new. In Bynon, Th. and Palmer, F.R. (eds.), Studies in the history of Western linguistics, Cambridge, pp. 56-68.

Simone, Raffaele. 1976. Sperone Speroni et l'idée de diachronie dans la linguistique de la Renaissance italienne. In Parret, H. (ed.), History of Linguistic Thought and Contemporary Linguistics, Berlin/New York, pp. 302-316.

Skytte, Gunver. 1990. Dall'Alberti al Fornaciari. Formazione della grammatica italiana. In Revue Romane, 25, 2, pp. 268-278.

Spanio, Michela L. Nicchetti. 1979. Accademie padovane nel Sei e nel Settecento. In Accademie e cultura. Aspetti storici tra Sei e Settecento. Biblioteca dell'edizione nazionale del carteggio di L.A. Muratori. Vol. V. Firenze, pp. 211-221.

Speroni, Sperone. 1989. Opere. Vol. I-V. Roma.

Stati, Sorin. 1976. Strumenti logici per la linguistica. Bologna.

Swiggers, Pierre. 1988. La clarté du francais: Examen historique et méthodologique. In Z. Phon. Sprachwiss. Kommunik.-forsch. (ZPSK), 41, 5, pp. 618-630 .

Tavoni, Mirko. 1986. The 15th-Century Controversy on the Language Spoken by the Ancient Romans: An inquiry into Italian humanist concepts of 'Latin', 'grammar', and 'vernacular'. In Ramat, P. et al. (eds.), The History of linguistics in Italy, Amsterdam/Philadelphia, pp. 23-50.

Tekavčić, Pavao. 1972. Grammatica storica dell'italiano. Vol. I-III. Bologna.

Povzetek

\section{ZAMISEL ZGODOVINE JEZIKA V SLOVNIČNIH DELIH BENEDETTA BUOMMATEIA}

Na splošno prevladuje mnenje, da je zgodovina jezika dokaj mlada jezikoslovna disciplina. Članek skuša pokazati, da so vprašanja o jezikovnih spremembah zanimala že renesančno dobo. Avtorica izhaja iz del italijanskega slovničarja Benedetta Buommateia (1581-1647), torej že iz dobe po Renesansi, in tehta mnenja in teorije o izvoru jezika in o vzrokih jezikovnih sprememb, kot so jih bili izrazili in postavili italijanski renesančni učenjaki. 